\title{
From Milliarcsecond Proper Motions to the Discovery of Dark Matter: Some Winning Cards still in the hands of Photographic Astronomy
}

\author{
J. Guibert \\ Centre d'Analyse des Images/MAMA, and Observatoire de Paris \\ (DEMIRM), 61 av. de l'Observatoire, 75014 Paris, France

\section{J. Souchay} \\ Centre d'Analyse des Images/MAMA, and Observatoire de Paris \\ (DANOF), 61 av. de l'Observatoire, 75014 Paris, France
}

\begin{abstract}
The large field of Schmidt plates allows monitoring of hundreds of thousands or even millions of stars, either for magnitude variations or for proper motions. CCD cameras benefit from the sensitivity, linearity, and easy interface to computers of detectors of increasing dimensions. However, especially for proper motion studies, there is no substitute to plates of first or intermediate epochs. In addition, the photographic plates, with their large sky coverage free of gaps, are irreplaceable for the accurate astrometry of optical counterparts of sources detected at other wavelengths.

We first introduce the subject by mentioning a few problems concerning the replacement of Schmidt plates by CCD detectors. After a brief survey of astronomical applications requiring accurate astrometry or massive processing in large fields, we develop in more detail the results obtained in proper motion studies by several groups using the MAMA system (Berger et al. 1991). The last part of this paper is devoted to the studies of stellar variability and to the EROS and DUO microlensing programmes. Our conclusion (not a surprise, we think), is that Schmidt telescopes with photographic facilities should remain available to the general astronomical community for at least a decade and probably more.
\end{abstract}

\section{Introduction: Operating CCD detectors in Large Fields}

The scientific programme of this colloquium clearly demonstrates that photographic emulsions not only are still used in a number of scientific projects, but will continue to play an important role even beyond the end of the millennium. At this time, several photographic sky surveys presently in progress will become available, not to speak of plates which, hopefully, will continue to be taken for special purposes, for example for galactic structure studies. Reasonable extrapolations of the increase in computer power and storage suggest that processing 
large sets of 12-14 inch plates digitised with a pixel size as small as 10 (or 5) $\mu \mathrm{m}$, already feasible at the present time, will then be a trivial matter.

On the other hand, the role of CCD cameras in astronomy is already important and continuously increasing. However, as developed for instance by Van Altena (1993) and Comte \& Surace (1993), a number of problems will have to be solved before CCDs can fully replace the plates.

A first class of difficulties will remain in the use of CCD detectors as long as the chips will be of modest size compared to Schmidt plates. For guided CCD exposures, in order to achieve a uniform system capable of being transformed into a standard astrometric system such as the PPM (Roeser 1990), it is necessary, when using "small" CCDs, to perform a corner to center overlap in the exposures, at the expense of telescope and data processing time. Using more CCDs or larger arrays in the focal plane, will be possible only with either a flattened optics, or a complex geometry of the detector. As a result, using the entire curved focal "plane" of classical Schmidt telescopes is not straightforward.

For drift scans, avoiding smearing out of the pixels imposes significant limitations on the size of the detector both in declination and right ascension, particularly for fields away from the celestial equator. These problems can be bypassed at the expense of various sophistications: for example, using several small chips displaced in declination (with some overlap problems), each with its own reading clock rate; using special driving modes of the telescope, etc.

\section{Astronomical Domains requiring accurate Astrometry in large Fields}

Schmidt plates can be scanned on fast and accurate microdensitometers like MAMA for identification, astrometry, and photometry of optical counterparts of sources detected at other wavelengths. Here we can mention, in particular, gamma and X-Ray emitting objects in studies of star forming regions (Feigelson et al. 1993 ), or binaries (Chevalier \& Ilovaisky 1990, Pakull et al. 1993).

The definition of an accurate reference system requires first a wide field to ensure that a sufficient number of good astrometric standards will be available to model the (plate or CCD image) geometry and, when no optical counterpart is detected, to calibrate a secondary astrometric catalogue. Combining the quality of Schmidt plates, their wide area, and the accuracy of MAMA, the PPM catalogue (Roeser 1990) leads to errors smaller than 0.3 arcsec ( 0.2 for the southern sky). For a machine of this class, a still better accuracy $(0.1$ arcsec $)$ will be achieved when the HIPPARCOS/TYCHO catalogues become available. Both the wide field and high quality astrometry are also of prime importance in slitless spectroscopy, for instance in studies of galaxies with active star formation, in particular for accurate determinations of radial velocities (Surace \& Comte 1994).

\section{Proper Motion Studies and Galactic Structure}

Several programmes combining the merits of Schmidt plates and MAMA have already produced proper motions with accuracy of the order of the 1-2 milli$\operatorname{arcsec} / \mathrm{yr}$ (Bienaymé et al. 1992, Bienaymé 1993, Chareton et al. 1993, Soubiran 
$1992 \& 1993$, Souchay \& Schilbach 1994). This accuracy, which favourably compares with the performances of the HIPPARCOS satellite, is due to:

1. a large number of plates per field ( 10 to 50$)$

2. the time baseline ( 20 to 40 years)

3. the micronic accuracy of the scans

4. a large number of background stars allowing accurate plate-to-plate polynomial transforms

5. a sufficient number of galaxies in the field, providing good absolute proper motions.

Obviously, here is a domain in which CCDs will not easily supersede the plates.

\section{Other Scientific Topics requiring Massive Processing of Large Fields}

Here, we would like to mention, among others, the systematic search for quasar candidates using objective prisms and multicolour photometry (Moreau et al. 1993), and the study of variable stars, for instance in the direction of the Galactic Bulge (Alard et al. 1993).

\section{Microlensing: the EROS and DUO programmes}

Gravitational amplification of the light emitted by background stars by massive objects located close to the line of sight, first predicted by Einstein (1936), is an interesting tool to probe the galactic populations (Paczinsky 1986 for the Galactic Halo, and 1991 for the Galactic Disk).

1. The flatness of rotation curves has important implications on the structure of the galactic haloes. Three microlensing events, likely to be caused by unseen objects of the Galactic Halo, have been reported in September 1993. One of them, found by the "MACHO" (Massive Compact Halo Objects) group (Alcock et al. 1993), was detected on CCD images. The two other ones (Aubourg et al. 1993), were detected by the French collaboration "EROS" (Experience de Recherche d'Objets Sombres), on ESO Schmidt plates digitized with the MAMA microdensitometer.

2. Another controversial issue is the disk mass density in the solar vicinity which should be consistent with the vertical acceleration $\mathrm{Kz}$. For instance, Bahcall (1984) suggests a large "missing mass" (up to $10^{-1} \mathrm{M}_{\odot} \mathrm{pc}^{-3}$ ), while, according to other authors (Bienaymé et al. 1987, Kuijken \& Gilmore 1989), there is no significant discrepancy between the required and the observed mass densities. It must also be pointed out that, even within $25 \mathrm{pc}$ from the Sun, our knowledge of the faint end of the luminosity function is 
very poor. Thus, any new information about the stellar density in the disk could seriously improve the situation. Paczinsky (1991), proposed to use also the microlensing of bulge stars to probe the density between the Sun and the Centre. The Warsaw group (Udalski et al. 1992, Udalski et al. 1993a), started the OGLE Project (Optical Gravitational Lensing Experiment), monitoring several hundreds of thousands stars on CCD images in small fields with a total area of about one square degree towards the Bulge, to search for microlensing candidates. Considering the astrophysical importance of any breakthrough in the puzzling "missing mass" problem, and the necessity to significantly increase the chance of detecting microlensing events, it was clear that all available means had to be used to detect as many candidates as possible. These considerations led a Paris-Besançon collaboration to submit a proposal to use the ESO Schmidt telescope to monitor several million bulge stars with a dense time sampling in the frame of another microlensing project named DUO (Disk Unseen Objects).

The DUO programme has been accepted, and will start in April 1994. In the meantime, the OGLE group (Udalski et al. 1993b, Udalski et al. 1994), announced the detection of the first four candidates microlensing events in the direction of the Bulge .

Since several millions stars can be followed in a single full 30 square degrees Schmidt field, one can be reasonably confident in the success of an operation in which 30 square degrees will be monitored. Plate taking facilities should therefore remain easily available to the astronomical community for projects of that size, together with the other types of programmes already mentioned in the preceding sections.

\section{Conclusion}

We have demonstrated, we think, that there are several domains of prime importance, in particular accurate astrometry, proper motion studies, and investigations like searches for microlensing candidates, in which CCDs will not easily supersede plates, at least in the near future. As a consequence, Schmidt telescopes with photographic facilities should remain available to the general astronomical community for at least a decade and probably more.

\section{References}

Alard C., Terzan A. \& Guibert J., 1993, The ESO Messenger, 73, 31

Alcock C. et al., 1993, Nature, 365, 621

Aubourg E. et al., 1993, Nature, 365, 623

Bahcall J. 1984, ApJ, 287, 926

Berger J., Cordoni J. P., Fringant A. M., Guibert J., Moreau O., Reboul H. \& Vanderriest C., 1991, A\&AS, 87, 389

Bienaymé O., Robin A. C. \& Crézé M., 1987, A\&A, 180, 94

Bienaymé O., 1993, A\&A, 278, 301 
Bienaymé O., Mohan V., Crézé M., Considère S. \& Robin A. C., 1992, A\&A, 253,389

Chareton M., Considère S. \& Bienaymé O., 1993, A\&AS, 102, 649

Chevalier C. \& Mlovaisky S. A. 1990, A\&A, 238, 163

Comte G. \& Surace C., 1994, in Astronomy from Wide-Field Imaging, Proc. IAU Symp. 161, in press

Einstein A., 1936, Science, 84, 506

Feigelson E. D., Casanova S., Montmerle T. \& Guibert J., 1993, ApJ, 416, 623

Garcia-Burillo S., Guélin M. \& Cernicharo J., 1993, A\&A, 274, 123

Kuijken K. \& Gilmore G., 1989, MNRAS, 239 ,605

Moreau O., Gosset E., Reboul H. \& Vanderriest C., 1994, in Astronomy from Wide-Field Imaging, Proc. IAU Symp. 161, in press

Paczinsky B., 1986, ApJ, 304, 1

Paczinsky B., 1991, ApJ, 371, L63

Pakull M. W., Motch C., Bianchi L., Thomas H.C., Guibert J., Beaulieu J. P., Grison P. \& Schaeidt S., 1993, A\&A, 278, L39

Roeser, S. 1990, in Inertial Coordinate System on the Sky, Proc. IAU Symp. 141, (Kluwer, Dordrecht), p. 469

Soubiran C., 1992, A\&A, 259, 394

Soubiran C., 1993, A\&A, 274, 181

Souchay J. \& Schilbach E., 1994, these proceedings

Surace C. \& Comte G., 1994, A\&A, 281, 653

Udalski A., Szymanski M., Kaluzny J., Kubiak M. \& Mateo M., 1992, Acta Astronomica, 42, 253

Udalski A., Szymanski M., Kaluzny J. \& Kubiak M., 1993a, Acta Astronomica, 43,69

Udalski A., Szymanski M., Kaluzny J., Kubiak M., Krzeminski W., Mateo M., Preston W. \& Paczinsky B., 1993b, Acta Astronomica, 43, 289

Udalski A., Szymanski M., Kaluzny J., Kubiak M., Krzeminski W. \& Mateo M., 1994, submitted to ApJ

Van Altena W. F., 1994, in Astronomy from Wide-Field Imaging, Proc. IAU Symp. 161, in press

\section{Discussion}

Drinkwater: Can you put any mass-limits on the lensing objects?

Souchay: It depends on the experiment. With the Schmidt plates programme, the mass of the stars which should be detected range theoretically between $10^{-4}$ and $1 \mathrm{M}_{\odot}$. For the CCD programme, the range is between $10^{-7}$ and $10^{-3} \mathrm{M}_{\odot}$. The effect of the impact parameter should extend these limits. 
Padmanabhan: Do you have more detailed results of any statistical analysis of the MACHO detection events? For example, has your team done a maximumlikelihood type analysis for the mass of the lensing candidate?

Souchay: Simulations have been carried out which were based on the observed flat rotation curve out to the LMC. The estimation of the mass of the lensing candidates is uncertain due to the lack of knowledge about their distance and speed. It ranges between $10^{-2}$ and $1 \mathrm{M}_{\odot}$. 